\title{
Les Palestiniens et le conflit syrien. Parcours de réfugiés en quête d'asile au Sud-Liban
}

The Palestinians and the Syrian Conflict. Itineraries of Refugees Seeking Asylum in South Lebanon

Los palestinos y el conflicto sirio. Itinerarios de refugiados solicitando asilo en el sur del Líbano

\section{Kamel Doraï}

\section{(2) OpenEdition \\ Journals}

\section{Édition électronique}

URL : https://journals.openedition.org/remi/7392

DOI : $10.4000 /$ remi.7392

ISSN : $1777-5418$

\section{Éditeur}

Université de Poitiers

\section{Édition imprimée}

Date de publication : 1 décembre 2015

Pagination : 103-120

ISBN : 979-10-90426-26-9

ISSN : 0765-0752

\section{Référence électronique}

Kamel Doraï, « Les Palestiniens et le conflit syrien. Parcours de réfugiés en quête d'asile au SudLiban », Revue européenne des migrations internationales [En ligne], vol. 31 - n³ et 4 | 2015, mis en ligne le 01 décembre 2018, consulté le 15 avril 2022. URL : http://journals.openedition.org/remi/7392 ; DOI : https://doi.org/10.4000/remi.7392 


\section{Les Palestiniens et le conflit syrien. Parcours de réfugiés en quête d'asile au Sud-Liban}

\section{Kamel Doraï ${ }^{1}$}

\section{Introduction}

Depuis la chute du régime de Ben Ali enTunisie en janvier 2011, les révolutions arabes ont occupé le devant de la scène médiatique et ont eu tendance à marginaliser la question palestinienne, pourtant longtemps centrale dans le paysage politique arabe (Dot-Pouillard, 2012). Le conflit syrien, et plus particulièrement le siège du camp palestinien de Yarmouk dans la banlieue de Damas à partir de décembre 2012, a rappelé qu'elle était toujours d'actualité. Le 31 janvier 2014, I'UNRWA $^{2}$ diffuse une photographie ${ }^{3}$ qui montre des milliers de Palestiniens, dans une rue bordée d'habitations détruites par les bombardements, convergeant vers un point de distribution d'aide alimentaire, après des semaines de siège de l'armée syrienne. Alors que ce camp comptait près de 150000 réfugiés palestiniens avant 2011, on estime que seuls 18000 y résident toujours en 2015. Au total, ce sont 280000 Palestiniens (sur les 520000 enregistrés auprès de I'UNRWA en Syrie ${ }^{4}$ ) qui ont été contraints de fuir leurs lieux de résidence ${ }^{5}$.

La situation particulière des Palestiniens doit être remise dans le contexte d'une Syrie en proie à un violent conflit qui a donné naissance à l'un des mouvements de réfugiés et de déplacés internes les plus massifs au Moyen-Orient depuis la Seconde Guerre mondiale. Le Haut Commissariat pour les Réfugiés des Nations unies (HCR) estime que plus de 4 millions de Syriens ont quitté leur pays depuis le début de la crise.

1 Chercheur CNRS, IFPO, UMIFRE 6/USR 3135, MAEDI/CNRS, 3 I. Zahri Street, BP 830413Zahran 11183, Amman, Jordanie ; mkdorai@univ-poitiers.fr

2 L'UNRWA (United Nations Relief and Works Agency for Palestine Refugees in the Near East) est I'Office de secours et de travaux des Nations unies pour les réfugiés de Palestine dans le Proche-Orient. II a été créé en 1949 par une résolution de I'Assemblée générale des Nations unies.

3 Cf. URL : http://www.unrwa.org/crisis-in-yarmouk, consulté le 31/08/2015.

4 Cf. UNRWA (2014) In figures, 2 p. [en ligne] consulté le 31/08/2015. URL : http://www. unrwa.org/sites/default/files/in_figures_july_2014_en_06jan2015_1.pdf

5 Cf. UNRWA (2014) Syria Regional Crisis Emergency Appeal 2015, 36 p., [en ligne] consulté le 14/06/2015. URL : http://www.unrwa.org/sites/default/files/syria_regional crisis_emergency_appeal_2015_english.pdf 
Tableau 1 : Réfugiés syriens enregistrés au HCR au Moyen-Orient (août 2015)

\begin{tabular}{|l|l|}
\hline \multicolumn{1}{|c|}{ Pays d'accueil } & \multicolumn{1}{c|}{ Réfugiés enregistrés } \\
\hline Turquie & 1938999 \\
\hline Liban & 1113941 \\
\hline Jordanie & 629245 \\
\hline Irak & 250408 \\
\hline Égypte & 132375 \\
\hline Total & $\mathbf{4} 064968$ \\
\hline
\end{tabular}

Source : Inter-agency Information Sharing Portal, Syria Regional Refugee Response, [en ligne] consulté le 31/08/2015. URL : http://data.unhcr.org/syrianrefugees/regional.php

Ce conflit a également de profondes conséquences sur l'exil forcé, souvent moins médiatisé, d'une partie de la population palestinienne de Syrie. Plus de 70000 personnes (soit 13,5\% de la population palestinienne totale enregistrée à I'UNRWA) se sont réfugiées dans les pays voisins. Près de 45000 réfugiés enregistrés ont quitté la Syrie pour le Liban, 15000 pour la Jordanie et 9000 pour l'Égypte (Doraï, 2015).

À l'image de leurs homologues syriens, la majeure partie des Palestiniens a trouvé refuge dans des pays du Moyen-Orient. À l'échelle régionale, la géographie de l'exil palestinien diffère cependant, puisque le Liban accueille plus de la moitié d'entre eux, contre un quart des Syriens. Comment expliquer ce tropisme vers un pays qui pratique l'une des politiques les plus discriminantes à l'égard des Palestiniens ? L'effet combiné de l'éclatement des familles entre les différents pays de la région depuis 1948, des circulations transfrontalières qui se sont développées à l'échelle régionale, comme du rôle de la diaspora dans la structuration des mobilités actuelles permet en partie de répondre à cette question. Une autre partie de la réponse se trouve dans la politique migratoire libanaise - et la capacité de l'État libanais à la mettre en œuvre - qui a évolué depuis le début de la crise, ouverture dans un premier temps, puis durcissement par la suite.

Le conflit syrien s'inscrit dans la continuité des bouleversements que connaît le Moyen-Orient depuis le début des années 1990. Les États actuels, héritages de la colonisation européenne, sont ébranlés dans leur unité territoriale. Les Kurdes affirment leur autonomie dans le nord de I'Irak, tandis que de larges zones à dominante sunnite échappent au contrôle de l'État central et sont sous le contrôle de l'État islamique. La Syrie subit aujourd'hui un destin semblable. Quelle sera alors la place dans ces reconfigurations nationales pour des Palestiniens, réfugiés et apatrides ? Pour reprendre les termes de Malkki (1995: 496), ces derniers sont exclus de l'ordre national en construction aujourd'hui en Syrie, alors qu'ils étaient l'un des groupes palestiniens les mieux intégrés à leur société d'accueil. Leur exil est donc symptomatique de leur marginalisation en Syrie, sans grand espoir à court terme de pouvoir escompter regagner leur lieu de résidence d'avant la crise. 
Cet article repose sur deux séries d'enquêtes réalisées, en décembre 2013 et mai 2015, auprès de vingt-cinq familles ${ }^{6}$, dans les camps et groupements ${ }^{7}$ palestiniens autour de la ville de Tyr, et plus particulièrement le camp d'Al Buss, et les groupements de Nahr al Samir, Jall al Bahr et Chabriha, ainsi que plus ponctuellement d'autres quartiers au nord de l'agglomération de Tyr ${ }^{8}$. Certaines familles ont été enquêtées lors des deux séjours, afin de suivre l'évolution de leur situation socio-économique et de leur projet migratoire. Les éléments recueillis ont été remis dans leur contexte, grâce à de multiples séjours courts dans la région depuis le déclenchement de la crise syrienne en 2011 et mis en perspective avec l'observation, depuis le milieu des années 1990, des mobilités palestiniennes entre le Liban et la Syrie. La population enquêtée appartient dans sa très large majorité aux classes sociales défavorisées en Syrie. Elle est constituée principalement d'ouvriers journaliers et d'employés peu qualifiés du secteur privé et public ${ }^{9}$.

Après avoir analysé les changements liés au déclenchement du conflit syrien pour la population palestinienne de Syrie et les contraintes auxquelles les réfugiés palestiniens doivent faire face au Liban, une analyse des parcours migratoires chaotiques de ces réfugiés sera proposée à partir des enquêtes de terrain réalisées dans la région deTyr.

\section{De Syrie au Liban, des réfugiés palestiniens en quête d'asile}

Le Liban, comme les autres pays de la région, n'est pas signataire de la Convention de Genève de 1951 sur les réfugiés (Zaiotti, 2006) ${ }^{10}$. Seuls les Palestiniens sont reconnus comme réfugiés dans l'État où ils ont leur résidence habituelle et lorsqu'ils sont inscrits auprès de I'UNRWA. Les États de la région n'ont donc pas de système d'asile national. C'est le HCR qui met en place des

\footnotetext{
6 Ces enquêtes ont été réalisées dans le cadre de I'ANR MOBGLOB. Je remercie Jaber Abu Hawash de l'aide précieuse qu'il m'a apportée pour rencontrer les familles palestiniennes de Syrie et pour m'avoir donné les éléments de contexte utiles à la compréhension des enjeux locaux liés à la présence de ce nouveau groupe de réfugiés au Sud-Liban.
}

7 La distinction entre camp et groupement repose sur le statut juridique de ces espaces. Alors que le camp est un espace reconnu par l'État d'accueil, dont la gestion est confiée à I'UNRWA, le groupement (tajamouaa en arabe) est une zone d'habitation informelle créée par les réfugiés eux-mêmes qui ne relève pas du mandat de I'UNRWA.

8 La population palestinienne est concentrée sur le littoral libanais autour des principales villes, Tyr, Saïda, Beyrouth et Tripoli, mais aussi, dans une moindre mesure, dans la plaine de la Beqaa autour des villes de Baalbek et de Zahlé. Le Sud-Liban avec les villes de Saïda et Tyr concentre à lui seul près de la moitié de la population enregistrée dans les camps du Liban. Autour de Tyr, de nombreux groupements informels se sont développés. Ce sont les réfugiés palestiniens d'origine bédouine qui se sont installés dans ces espaces plutôt que dans les camps de réfugiés dès leur arrivée au Liban entre 1948 et 1956. Cet espace est aussi I'un des principaux foyers de départ vers les pays d'Europe du Nord depuis le milieu des années 1970 (Doraï, 2006).

9 Les classes sociales plus favorisées qui ont trouvé également asile au Liban n'ont pas été enquêtées dans le cadre de cette recherche. Ces réfugiés ont accès à des ressources (financière, familiale, professionnelle) plus importantes, qui facilitent leur mobilité (Van Hear, 2014).

10 Israël, I'Égypte et la Turquie sont signataires de la convention, mais avec des réserves, ce qui limite l'application de la convention. 
procédures d'asile et collabore avec les autorités des pays concernés avec la signature d'un Memorandum of understanding, qui précise le mandat du HCR (Kagan, 2011 : 9). Les Palestiniens sont de facto hors du champ d'application de I'asile conventionnel dans les pays et territoires où exerce I'UNRWA (Feldman, 2012). Les différents pays ayant déjà accueilli des réfugiés palestiniens en 1948 et 1967 pour certains - considèrent que la responsabilité de leur accueil incombe à la communauté internationale. La Jordanie a par exemple fermé ses frontières aux réfugiés palestiniens de Syrie en 2013, par crainte d'être considérée comme une patrie de substitution. Si la majorité des régimes arabes affiche un soutien appuyé à la cause palestinienne, et en particulier au droit au retour des réfugiés, le statut précaire ${ }^{11}$ de ces derniers, en particulier en situation de crise, montre le peu de soutien dont ils bénéficient en réalité. Priment avant tout les intérêts politiques, économiques et sécuritaires des États d'accueil en fonction de la conjoncture régionale et internationale. $C^{\prime}$ est en ce sens que le changement politique en Syrie qui intervient en 2011 va bouleverser la situation des réfugiés palestiniens qui y résidaient depuis 1948. Jusque-là, la Syrie s'affichait comme le leader arabe de la résistance face à Israël et de ce fait soutien de la cause palestinienne. La révolution va remettre en cause ce schéma, les Palestiniens étant perçus par le régime comme potentiellement source d'instabilité, remettant en cause un long processus d'intégration, pour reprendre l'expression de Brand (1988).

\section{1, une rupture pour les Palestiniens de Syrie}

Le statut des Palestiniens depuis leur exil en 1948 dépend du pays dans lequel ils se sont installés. La Syrie à cet égard fait figure d'exception dans la région (Brand, 1988 ; Fadhel, 2012 ; Napolitano, 2012). Suite à une série de lois proclamées entre 1949 et 1956, les réfugiés palestiniens bénéficient en Syrie des mêmes droits que les citoyens syriens, à l'exception de la nationalité et de restrictions dans I'accès à la propriété. Ils jouissent par exemple pleinement d'un accès à l'éducation et au marché du travail (incluant la fonction publique). C'est également le seul pays où les Palestiniens doivent effectuer leur service militaire, dans une unité spéciale, bien qu'ils ne soient pas citoyens. Ce traitement est en partie lié au fait que, contrairement à la Jordanie, mais aussi au Liban dans une moindre mesure, la population palestinienne n'a jamais représenté plus de $3 \%$ de la population syrienne. D'autant plus qu'à leur arrivée, la Syrie ne manquait pas de ressources naturelles et ne connaissait pas de difficultés économiques particulières. De façon générale, Brand relève que cette égalité de traitement a limité le développement d'organisations de masses palestiniennes - comme les syndicats - distinctes de celles de leurs pays d'accueil (Brand, 1988 ; Takkenberg, 1998).

11 La précarité du statut des réfugiés palestiniens dans leurs différents pays d'accueil repose en partie sur la capacité ou non de I'UNRWA à assurer leur protection, particulièrement en situation de conflit. Si I'UNRWA dispose aujourd'hui formellement d'un mandat de protection, sa capacité à le mettre en place est de facto limitée par les autorités des différents pays d'accueil, et se cantonne le plus souvent à l'assistance humanitaire et au plaidoyer en faveur des réfugiés. La collaboration entre le HCR et I'UNRWA dans ce domaine, si elle a pu montrer sa relative efficacité pour les Palestiniens fuyant I'Irak en 2003, ne permet pas aujourd'hui de répondre aux conséquences de la crise syrienne pour les Palestiniens contraints de s'exiler dans les pays voisins (Erakat, 2014 ; Kagan, 2009). Les réfugiés palestiniens de Syrie sont par exemple dépendants des autorités syriennes pour sortir du pays. Le gouvernement syrien est le seul habilité à leur délivrer un document de voyage valide. 
L'année 2011 représente donc une rupture pour les Palestiniens de Syrie. Ils se voient renvoyés à leur statut d'apatrides, privés de protections, dépendant de l'aide humanitaire et contraints pour certains à chercher asile dans un des pays frontaliers, à l'instar des Palestiniens qui ont dû fuir le Koweït en 1990-1991 ou I'Irak suite à la chute du régime de Saddam Hussein en 2003 (Doraï et Al Husseini, 2013). Ce basculement ne s'est pas opéré de façon uniforme dans l'ensemble de la Syrie et, à bien des égards, n'a pas concerné uniquement la population palestinienne. Aujourd'hui, c'est une large frange du peuple syrien qui subit les conséquences du conflit. Cependant, la population palestinienne s'est trouvée très rapidement entraînée dans le soulèvement et prise pour cible par le régime syrien, mais aussi par des groupes insurgés. La géographie du conflit joue un rôle majeur dans l'exposition aux combats et destructions. Les premiers camps touchés sont ceux qui se situent dans les principales zones de combat ou d'intérêt stratégique. Le camp d'Al Ramel à Lattaquieh a par exemple été pris pour cible dès le mois d'août 2011. A contrario, les camps de Yarmouk ou de Jaramana, tous deux dans la banlieue sud de l'agglomération damascène, vont servir d'espace refuge tant pour les Syriens que pour les Palestiniens fuyant les combats. Le camp de Yarmouk, touché plus tardivement par les combats, arrive à préserver une certaine neutralité jusqu'en décembre 2012 (Napolitano, 2015). L'extension des combats dans les quartiers périphériques de la capitale syrienne, où résident la majeure partie des Palestiniens, va les contraindre à fuir vers des régions plus calmes, le plus souvent celles tenues par le régime d'Al Assad, ou vers les pays frontaliers.

Le positionnement politique de certaines factions palestiniennes dans le conflit syrien a contribué à fragiliser la position des réfugiés, particulièrement vis-à-vis du régime de Bachar Al Assad. Alors que le chef du Bureau politique du Hamas, Khaled Mechaal, résidait en Syrie depuis 1999, les membres de sa direction vont quitter Damas en 2012 (Dot-Pouillard, 2012). Certaines factions palestiniennes soutiennent le régime de Bachar Al Assad, mais disposent de peu de relais dans la population palestinienne. La situation des Palestiniens en Syrie oscille entre des formes de contestation individuelle et des mouvements plus structurés d'opposition tant au régime qu'à certaines franges du leadership palestinien en Syrie (Napolitano, 2011 et 2012), même si une majorité de réfugiés ne prend pas part aux combats de façon active que ce soit pour ou contre le régime syrien, ce qui est confirmé par les témoignages recueillis au Sud-Liban.

\section{Le Liban, un refuge précaire}

Le Liban est connu dans la région pour être l'un des États où le statut des réfugiés palestiniens est le moins enviable et sujet à de nombreuses restrictions (Al-Natour, 1997). En 1995, quand le régime de Kadhafi expulse les Palestiniens, ou en 2003 quand ils sont contraints de quitter I'Irak suite à la chute du régime de Saddam Hussein, le Liban refuse I'entrée sur son sol de réfugiés palestiniens venus de Libye ou d'Irak. La crise syrienne force le Liban à revenir temporairement sur cette politique, puisqu'il est actuellement le principal pays d'accueil de cette population. Outre l'expression d'une certaine solidarité vis-à-vis des réfugiés contraints de quitter la Syrie, les divisions de la classe politique libanaise quant au conflit syrien sont en partie responsables de la mise en place tardive des restrictions à l'entrée des réfugiés palestiniens. 
Si le Liban est le deuxième pays d'accueil des réfugiés syriens, il est le premier pour les réfugiés palestiniens de Syrie (Doraï, 2015). Le nombre de réfugiés palestiniens de Syrie présents au Liban reste cependant difficile à évaluer. II faut rappeler que les réfugiés palestiniens ne dépendent pas du HCR, mais de I'UNRWA à condition qu'ils se trouvent dans I'une des cinq régions ${ }^{12}$ où officie I'agence onusienne (Doraï et Hanafi, 2003). Selon I'UNRWA (2015), près de 45000 Palestiniens de Syrie seraient entrés au Liban depuis de la crise ${ }^{13}$. Ces chiffres sont cependant à prendre avec précaution, ils ne résultent pas d'un recensement de la présence effective des réfugiés palestiniens de Syrie au Liban, mais d'un enregistrement administratif des réfugiés ayant franchi la frontière entre les deux pays. Tant que la frontière était ouverte, de nombreux Palestiniens se rendaient de façon temporaire au Liban, pour ensuite retourner en Syrie selon l'évolution de la situation dans les camps et/ou villes de résidence habituelle. Depuis le mois de mai 2014, le Liban a fermé ses frontières à cette catégorie de réfugiés avec pour conséquence la fin de la circulation entre les deux pays. Des familles se sont donc retrouvées, de fait, séparées. Les frontières libanaises restent cependant assez poreuses. Des entrées illégales sur le territoire libanais ont donc lieu, soit pour contourner la fermeture de la frontière, soit parce que certains réfugiés considérés comme des opposants par le régime syrien sont privés de documents d'identité ou ne peuvent les produire. Par ailleurs, des Palestiniens dont une partie de la famille réside au Liban viennent se réfugier chez leurs proches ou vivent en dehors des camps ${ }^{14}$, sans s'inscrire auprès de I'UNRWA.

\section{Le durcissement progressif de la politique migratoire libanaise}

Face à la crise syrienne, la politique migratoire libanaise ne fait pas figure d'exception dans la région. Les politiques des États voisins envers les réfugiés de Syrie ont changé au fil du temps allant d'une ouverture des frontières à une fermeture de plus en plus rigoureuse, avec l'instauration de visas pour les ressortissants syriens dans l'ensemble des pays frontaliers. Au Liban, c'est en 2014 qu'un tournant est opéré avec la remise en cause d'un accord bilatéral concernant l'emploi de la main-d'œuvre syrienne en vigueur depuis 1994 (Longuenesse, 2015), alors que dans le même temps, les mobilités de main-d'œuvre et de réfugiés s'intensifient. Si le conflit en Syrie a entraîné la migration forcée de plusieurs centaines de milliers de réfugiés, les migrations économiques n'ont cependant pas disparu entre les deux pays. Près de 400000 Syriens travaillaient au Liban avant 2011 (Chalcraft, 2009), la plupart y sont restés ; certains ne pouvant pas rentrer chez eux sont devenus de fait des réfugiés et ont été s'inscrire au HCR.

Au début de la crise syrienne, la Sûreté générale du Liban, qui est en charge de la gestion des modalités d'entrée et de séjour des étrangers, a pris des mesures spécifiques pour faciliter l'entrée des réfugiés palestiniens de Syrie sur le sol libanais. Le Liban appliquait jusqu'en 2011, comme la majeure partie

12 Bande de Gaza, Cisjordanie, Jordanie, Syrie, Liban.

13 Cf. UNRWA (2015) UNRWA response and services to PRS in Lebanon, 43, 2 p. [en ligne] consulté le 26/05/2015. URL : http://www.unrwa.org/sites/default/files/unrwa_ response_to_palestine_refugees_from_syria_in_lebanon_43.pdf

14 Près de $50 \%$ des réfugiés palestiniens du Liban vivent en dehors des camps. 
des pays arabes, des mesures restrictives pour limiter l'entrée des Palestiniens. Les autorités justifient cette politique par la volonté de limiter les " risques" d'installation de Palestiniens non enregistrés au Liban et de voir augmenter leur nombre dans un contexte où le refus de l'implantation des réfugiés palestiniens fait la quasi-unanimité dans la classe politique libanaise.

Jusqu'en 2013, dans un but humanitaire, la Sûreté générale libanaise a cependant décidé d'assouplir les conditions d'entrée et de séjour des réfugiés palestiniens venus de Syrie. Ils devaient acquitter un droit d'entrée de 25000 livres libanaises (LL - environ $13 €$ ) par personne, le visa délivré ayant une validité de sept jours. Ils pouvaient renouveler leur permis de résidence pour une durée d'un mois pour 50000 LL (environ $26 €$ ). Ils devaient ensuite quitter le territoire avec la possibilité de renouveler la procédure. Pour ceux ayant dépassé la durée légale de résidence et qui se présentaient à la frontière pour regagner la Syrie une amende de 50000 LL leur était demandée ; cette mesure a été supprimée en septembre 2012. L'objectif de ces mesures était de limiter autant que possible l'installation sur le long terme de réfugiés palestiniens de Syrie au Liban, et de n'autoriser que des séjours de courtes durées qui ne donnent aucun droit spécifique, comme l'accès au marché du travail, à l'éducation ou à la santé. Concernant ces deux derniers domaines, c'est I'UNRWA qui prend en charge les réfugiés qui sollicitent une assistance.

Le coût de la procédure d'extension de visa est difficile à supporter pour de nombreuses familles qui deviennent donc illégales, même si la Sûreté générale ne semble pas procéder à beaucoup de vérifications. Ces familles s'installent donc fréquemment dans les camps de réfugiés palestiniens, souvent auprès de proches, de façon à échapper aux éventuels contrôles des autorités. Ces pratiques ont entraîné une augmentation des prix du logement dans les camps palestiniens, déjà soumis à une forte pression démographique.

À partir d'août 2013, les Palestiniens de Syrie devaient prouver qu'ils avaient des liens familiaux au Liban, ou bien qu'ils venaient pour un rendez-vous médical ou dans une ambassade. Transiter par le Liban était autorisé. Quelques exceptions à ces règles ont pu être observées, et ce à la discrétion de la Sûreté générale. Depuis le 3 mai 2014, face à l'afflux toujours plus important de réfugiés venus de Syrie, la Sûreté générale libanaise a durci considérablement les conditions d'entrée des réfugiés palestiniens de Syrie sur son sol ${ }^{15}$. En conséquence, aujourd'hui la majeure partie de ces réfugiés se voit refuser l'entrée au Liban.

Dans le même temps, les autorités libanaises ont mis en place des restrictions au renouvellement des visas de séjour pour les Palestiniens de Syrie déjà présents sur son sol. Nombreux sont ceux qui résident illégalement au Liban, limitant de fait leur mobilité, l'accès aux services et à l'assistance, ainsi qu'à des procédures administratives comme l'enregistrement des naissances. À plusieurs reprises, la Sûreté générale a appelé les Palestiniens de Syrie en infraction avec la législation sur le séjour à venir régulariser leur situation. Ceux qui se mani-

15 II faut relever qu'en décembre 2014, des mesures restrictives pour l'entrée sur le territoire libanais ont également été prises à l'encontre des réfugiés de nationalité syrienne. Les modalités d'entrée et de séjour ont été redéfinies en fonction de l'objet du séjour des ressortissants syriens, distinguant ainsi différentes catégories et types de visas. Cf. URL : http://www.general-security.gov.lb/results/0102031.pdf, consulté le 28/01/2015 (en arabe). 
festent se voient octroyer un visa de trois mois non renouvelable. La dernière régularisation remonte au 28 août $2014^{16}$. Par ailleurs, depuis la mise en place des restrictions à l'entrée, de nombreux Palestiniens de Syrie ont été reconduits sans leur consentement à la frontière syrienne, bien qu'ils risquaient d'être détenus à leur entrée en Syrie. Certains d'entre eux se trouvent de facto bloqués dans le no man's land entre les deux pays. Cette situation n'est pas sans rappeler celle des réfugiés palestiniens d'Irak, suite à la chute du régime de Saddam Hussein en 2003, ou celle des Palestiniens expulsés de Libye en 1995 (Doraï et Al Husseini, 2013).

\section{Des parcours migratoires chaotiques}

Les Palestiniens de Syrie qui arrivent au Liban rejoignent aujourd'hui un segment de la diaspora fortement marqué par l'émigration ${ }^{17}$, ce qui a de fortes implications tant sur les modalités de leur installation que sur la poursuite éventuelle de leur parcours, plus particulièrement à Tyr. Les groupements informels étalés le long de la côte, entre le fleuve Litani et le nord de la ville de Tyr, ont la particularité d'être habités en majorité par les Palestiniens issus de familles bédouines du nord de la Galilée. Elles se sont retrouvées dispersées suite à l'exode de 1948 entre les différents pays de la région, mais ont conservé des modes d'organisation sociale singuliers, qui les différencient des autres Palestiniens réfugiés. Même si le mode de vie de ces populations, qui se qualifient de Bédouins, a très fortement changé dans l'exil, elles ne pratiquent plus l'élevage, sont toutes devenues sédentaires et attachent une importance particulière à la préservation de leur singularité (Zureik, 2003 ${ }^{18}$ ). Elles essayent donc de reconstituer, par le biais de regroupements familiaux et claniques, une unité socio-spatiale distincte de celle des Palestiniens des villages et des villes. La référence à la tribu (aachira ou qabila ${ }^{19}$ ) est centrale dans le discours des réfugiés palestiniens de Syrie d'origine bédouine : elle joue notamment un rôle important pour les modalités d'installation et des connexions transnationales qui vont structurer les réseaux migratoires vers l'Europe du Nord.

\section{Une forte concentration dans les camps et groupements palestiniens déjà présents}

Comme leurs homologues installés de longue date au Liban, un peu plus de la moitié des réfugiés palestiniens de Syrie récemment arrivés se concentrent

16 Direction générale de la Sûreté générale libanaise, Régularisation de la situation des réfugiés palestiniens de Syrie résidant au Liban, 28/08/2014.

17 Selon une étude publiée en 2010 (Chaaban et al., 2010), sur les 425000 réfugiés palestiniens enregistrés par I'UNRWA au Liban, 260000 à 280000 résideraient sur le territoire libanais, les autres ayant émigré vers des pays tiers. Le taux d'émigration de ce groupe est donc très élevé puisqu'il concerne plus du tiers de la population concernée. Cette émigration repose sur des logiques plurielles liées aux différents conflits qui ont déchiré le Liban depuis 1975, les difficultés économiques et la marginalisation subies par les réfugiés palestiniens, comme les logiques diasporiques qui se développent depuis les années 1990 (Doraï, 2003).

18 Zureik évoque dans son article ce mode d'organisation socio-politique à propos du camp de Jerash en Jordanie. Une structuration similaire se retrouve dans les groupements palestiniens deTyr.

19 Le terme arabe aachira est d'usage au Liban, en Syrie, en Palestine et Jordanie, celui de qabila dans les pays du Maghreb. 


\section{Carte 1 : Répartitions des Palestiniens réfugiés de Syrie (PRS) enregistrés à l'UNRWA au Liban (février 2014)}

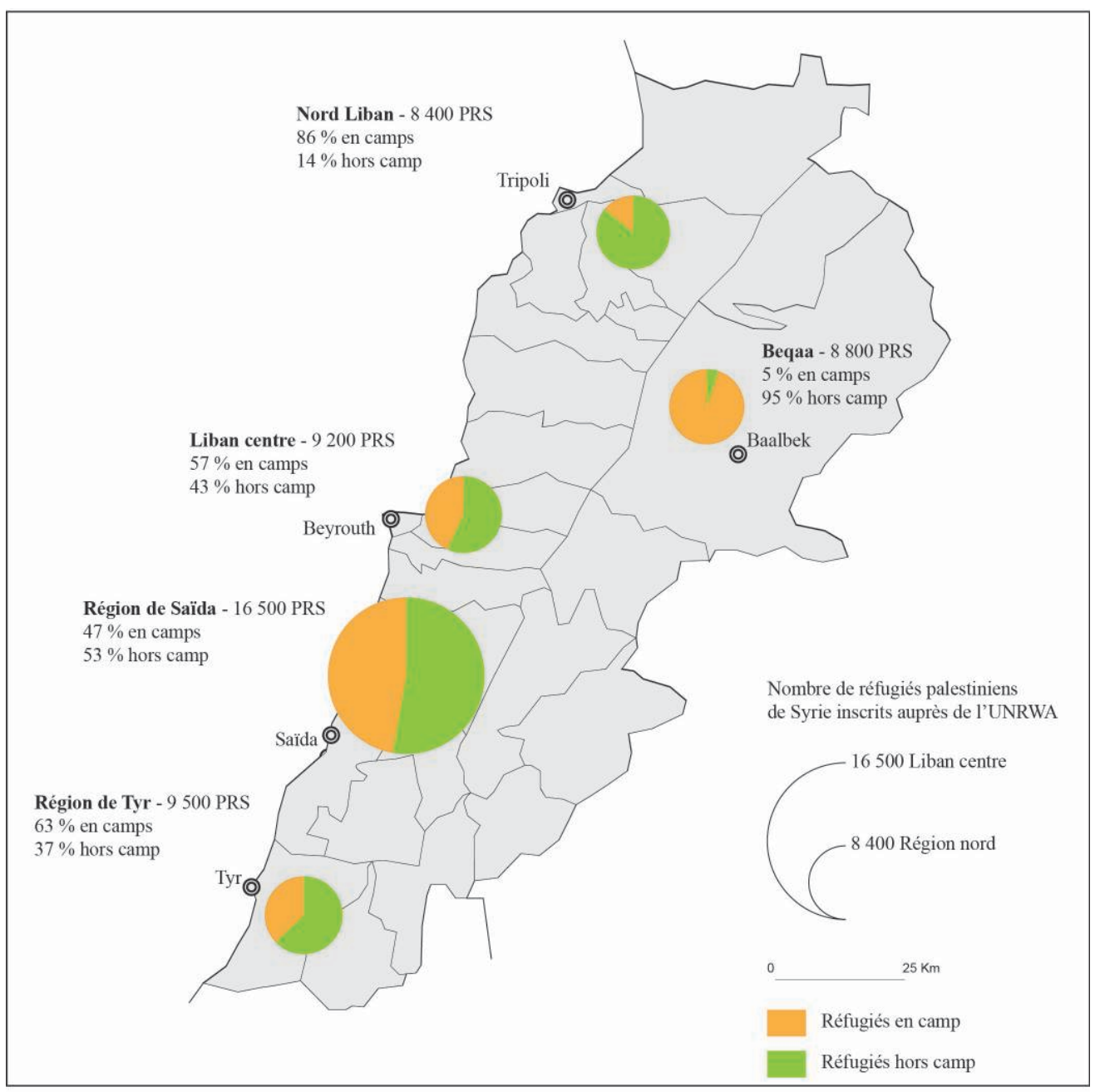

Source : UNRWA - Libanon field PRS in Libanon - geographical location (février 2014).

Crédits : M. Doraï - CNRS, Ifpo, 2015. 
dans I'un des douze camps de réfugiés déjà existants (Carte 1). Les autres s'installent dans des quartiers ou des groupements ruraux où la présence palestinienne est forte. Sa géographie n'a donc pas été fortement modifiée par les nouvelles arrivées, à l'exception d'une recrudescence des installations dans la plaine de la Beqaa, région frontalière de la Syrie et première étape pour ceux qui quittent Damas par la principale route qui la relie au Liban. En raison de la faible présence palestinienne dans cette région, $95 \%$ des Palestiniens ne vivent pas dans des camps, à l'instar de leurs homologues de nationalité syrienne qui y ont développé de très nombreux groupements informels (Knudsen, $2013: 32$ ).

On assiste donc à une densification des espaces habités par les Palestiniens et à une polarisation des migrations vers des quartiers périphériques des principales agglomérations littorales libanaises. Les logiques qui sous-tendent cette géographie de l'asile reposent sur plusieurs facteurs. Tout d'abord, les relations familiales préexistantes entre les deux groupes vivant au Liban et en Syrie. Quand le conflit s'est intensifié, les premiers réfugiés palestiniens à se rendre au Liban sont ceux qui y avaient des proches. Ils ont, pour la plupart, cherché dans ce pays voisin un asile temporaire. Ce n'est que lorsque la situation sécuritaire s'est détériorée de façon significative, qu'un nombre croissant de réfugiés ont stoppé leurs circulations entre les deux pays pour s'installer de façon durable au Liban. Les restrictions à la mobilité imposées par les autorités libanaises ont ensuite contribué à les stabiliser dans leur second pays d'accueil, de peur de ne pouvoir revenir s'ils se rendaient en Syrie, même pour une courte période.

La présence dans les camps et les principaux groupements palestiniens s'explique également par des raisons économiques. Ce sont des espaces de relégation où se concentrent de longue date les populations les plus défavorisées, qu'elles soient palestiniennes dans leur majorité, mais aussi migrantes venues d'horizons divers (Syriens, Soudanais, Bangladais, etc.). Le logement y est moins cher que dans d'autres quartiers des principales agglomérations libanaises. C'est le cas plus particulièrement à Beyrouth, où le camp de Chatila et ses abords - comme le quartier de Sabra - accueillent aujourd'hui une importante population palestinienne de Syrie, syrienne et asiatique. Si cet accueil repose sur des logiques de solidarité, il entraîne aussi des formes de concurrence entre les réfugiés palestiniens résidents et les nouveaux arrivants (Abou Zaki, 2015). À l'échelle du Liban, la sous-représentation dans l'agglomération beyrouthine s'explique par le niveau élevé des loyers dans la capitale, les ressources limitées des réfugiés les poussent à chercher des espaces d'accueil où les loyers et le coût de la vie sont moins élevés.

De plus, les camps palestiniens au Liban bénéficient d'un statut d'autonomie de fait. Les autorités libanaises ne pénètrent pas dans les camps, ni dans les groupements ruraux. Avec l'arrivée des réfugiés palestiniens de Syrie, ces lieux deviennent des espaces refuge pour des populations sans statut et vivant dans l'illégalité : les Palestiniens peuvent $s^{\prime} y$ installer sans risquer d'être arrêtés ou déportés vers la Syrie. Ils peuvent également y exercer une activité professionnelle, alors que cela leur est interdit sur le reste du territoire libanais. 


\section{De la circulation transfrontalière à l'asile}

Depuis 2011, les parcours des réfugiés reposent en partie sur des circulations transnationales mises en place ces dernières décennies entre le Liban et la Syrie. Malgré des restrictions, des circulations de Palestiniens de Syrie vers le Liban ont réussi à se développer.

Originaire de Haïfa, la famille de Jalal ${ }^{20}$ est installée à Tyr depuis le début des années 1950. Comme c'est souvent le cas, l'exode de 1948 a conduit à la séparation de la famille entre les différents pays de la région. Trois sœurs, ayant perdu leurs parents à cette période, se sont installées l'une à Tyr (Liban), la seconde à Alep (Syrie) et enfin une troisième à Amman (Jordanie). Les relations familiales entre les sœurs installées au Liban et en Syrie sont restées fortes, entretenues par de fréquentes visites. La deuxième génération, née en exil, renforce ces liens par le mariage entre les deux branches de la famille dans les années 1990 et au début des années 2000. Deux sœurs nées en Syrie se marient chacune avec un cousin au Liban, et une fille née au Liban épouse un de ses cousins de Syrie.

Parallèlement à ce renforcement des liens familiaux par les diverses stratégies matrimoniales, des membres de la parentèle sont venus au Sud-Liban pour travailler comme ouvriers saisonniers, comme le faisaient de nombreux travailleurs syriens à cette époque. Ces migrations ont contribué à créer un espace de circulation transfrontalier dense. Ces familles hébergent leurs proches et facilitent leur insertion sur le marché de l'emploi. Les liens se sont ainsi consolidés à travers les générations. Ces mobilités ne concernent pas les catégories sociales de Palestiniens les plus défavorisées en Syrie, car les coûts induits par cette migration sont élevés. À la différence de la période post-2011 qui touche toutes les catégories sociales, dans les années 1990, ce sont donc plutôt des Palestiniens relativement qualifiés qui circulent.

Cet espace migratoire structuré par un large réseau familial, qui concerne particulièrement les Palestiniens disposant d'un certain capital, a été mobilisé à nouveau, mais il s'est élargi. Parmi les familles interviewées, seules trois s'étaient déjà rendues au Liban avant la crise syrienne. Ceci est particulièrement vrai pour les réfugiés palestiniens d'origine bédouine. Leur niveau socio-économique en Syrie ne leur permettait pas d'entreprendre un voyage au Liban. Cette absence de circulation ne signifie pas pour autant une absence de liens symboliques qui s'appuient sur la connaissance des familles et de leur dispersion depuis 1948. Cette mémoire familiale (ou plus largement tribale dans le cas spécifique des Palestiniens d'origine bédouine) permet de savoir où se trouvent les proches et de garder ainsi le contact, le plus souvent par téléphone. Les mariages et décès - en dehors des périodes de crise - sont aussi l'occasion d'échanger et de réactiver les liens sans même se déplacer.

\section{Le rôle structurant de la diaspora en situation de crise}

Le conflit syrien a contribué à redessiner la géographie de la diaspora palestinienne. Avant 2011, les Palestiniens de Syrie n'entretenaient que peu de relations avec leurs homologues d'Europe du Nord. Leur départ de Syrie les a

20 Les prénoms utilisés dans cet article sont des pseudonymes. 
forcés à activer, à partir du Liban, des réseaux familiaux, villageois ou tribaux ${ }^{21}$ et à développer ainsi une forme de "transnationalisme forcé ", selon Smith (2002), qui s'organise dans un contexte de crise autour de réseaux de solidarité à l'initiative des réfugiés palestiniens qui se trouvent dans les différents pays où ils sont accueillis.

Les réseaux de la diaspora palestinienne qui se sont construits dès les années 1980 entre le Liban, I'Europe du Nord et les pays du Golfe sont aujourd'hui très actifs et contribuent au dessin d'une géographie de l'exil des Palestiniens de Syrie. Une famille rencontrée en décembre 2013 dans le groupement informel palestinien de Chabriha, au nord de la ville deTyr, donne un exemple de ces liens internationaux. Habitant du quartier de Babila dans la banlieue sud de Damas, la famille a fui un quartier touché par les combats et les destructions importantes. Le mari, cantonnier dans le secteur public, a d'abord craint de quitter la Syrie, de peur d'être considéré comme appartenant à I'opposition. Alors que tous les fonctionnaires en Syrie, syriens ou palestiniens, doivent obtenir une autorisation de sortie du territoire pour se rendre à l'étranger, ce chef de famille quitte son quartier pour protéger sa famille en espérant que la situation s'améliore. Mais alors que la situation s'aggrave et que la stigmatisation dont les Palestiniens sont l'objet, accusés par le régime de soutenir l'opposition armée, se fait dangereuse, il décide d'envoyer son épouse et ses enfants au Liban dans le but de les rejoindre ultérieurement, ce qu'il va faire après plusieurs voyages entre Damas etTyr. Après un an de présence au Liban, il est dans l'impossibilité de renouveler son visa de résidence. II se retrouve donc à l'instar de nombreux Palestiniens dans une situation d'illégalité.

II est dans un premier temps logé dans sa famille, dans l'attente de trouver une solution plus pérenne. Puis il contacte des parents qui résident au Danemark et dans les pays du Golfe pour solliciter une aide. Ceux-ci réussissent à trouver un autre logement pour la famille dans le groupement, au dernier étage d'un immeuble en construction. L'argent qu'ils envoient va permettre l'installation mais surtout l'achèvement des travaux de l'appartement.

Alors que les constructions ont très longtemps été interdites par les autorités libanaises dans les camps officiels et les groupements informels, un assouplissement temporaire des contrôles a permis à de nombreux Palestiniens d'ajouter des étages aux bâtiments déjà existants. Ces groupements du Sud-Liban ont connu une très forte émigration dans les années 1980 et 1990 (Doraï, 2003) et de nombreuses familles, qui en sont originaires, résident et travaillent aujourd'hui soit en Europe (Suède, Danemark, Allemagne pour la majorité), soit dans les pays du Golfe. Elles maintiennent des liens forts avec leurs lieux de départ et contribuent au développement des constructions dans les groupements. Et cela, pour deux raisons : améliorer les conditions de vie de leurs proches restés au Liban et disposer d'un appartement où venir passer des vacances en été ou pour les fêtes. Ces logements vacants sont aujourd'hui occupés par des réfugiés palestiniens de Syrie. On assiste à la mise en place de formes d'entraide qui mettent en lien des réfugiés palestiniens du Liban, ceux installés dans des pays tiers et les nouveaux réfugiés venus de Syrie.

21 Les populations réfugiées mobilisent les réseaux de la diaspora dans des contextes contraints pour pallier la précarité de leur statut (Van Hear, 2006 ; Wahlbeck, 2002). 
Alors que les connexions entre ces espaces au Sud-Liban et les pays scandinaves demeurent très fortes, cette famille a pu quitter le Liban au premier semestre 2014 pour rejoindre le Danemark et y demander l'asile. II est intéressant de noter que préalablement à la crise, les différentes personnes qui se sont mobilisées ne se connaissaient pas toutes directement ; c'est le cas de celles qui résidaient en Syrie et de celles qui résidaient au Liban. Ces réseaux de solidarité à l'échelle de ce que I'on peut qualifier de grande diaspora (incluant les espaces hors du Moyen-Orient) permettent donc aux Palestiniens en quête d'asile de trouver dans un premier temps un refuge temporaire dans un pays voisin (ici le Liban), puis ils facilitent la réinstallation vers un pays tiers (ici le Danemark).

\section{Un asile qui dure. De la précarité à la marginalisation}

L'enlisement du conflit en Syrie et les restrictions à la mobilité des Palestiniens par les autorités libanaises contribuent à l'installation durable des réfugiés palestiniens de Syrie dans leur second pays d'accueil. Ceux qui ne peuvent espérer émigrer vers un pays tiers se retrouvent dans une impasse, car ils ne peuvent pas non plus retourner en Syrie. Leur situation se traduit par une paupérisation et une marginalisation croissante.

Rencontré une première fois en décembre 2013, un couple de Palestiniens originaires du camp de Yarmouk a quitté la Syrie en décembre 2012. Le mari était électricien à son compte avec un niveau de revenu assez faible. Son épouse, sans emploi, était enceinte à leur arrivée au Liban. Deux raisons les ont poussés à choisir ce pays alors qu'ils ne le connaissaient pas : la possibilité de s'y rendre et I'accueil attendu. À cette époque, c'était le seul pays permettant aux Palestiniens ne disposant que d'une carte d'identité (et sans documents de voyage) d'entrer légalement sur son territoire. De plus, l'épouse avait un oncle à Tyr qui offrait son aide, bien qu'elle ne l'ait jamais rencontré. Ils arrivent donc directement à Tyr depuis Damas. Leurs proches, aux ressources très limitées, ne sont ni en mesure de les héberger faute de place, ni de les aider financièrement. Ils leur trouvent cependant un logement dans un groupement palestinien. Le couple paye 200 USD par mois pour une pièce et une cuisine. Lors de cette première rencontre, ils possédaient une carte de séjour qui leur permettait de résider légalement sur le sol libanais. Le mari occupait temporairement un emploi journalier comme manutentionnaire dans le secteur informel de la construction.

Lors de notre deuxième entrevue en mai 2015, leur situation s'est dégradée, car ils n'ont pas pu renouveler leur carte de résidence. Cette situation limite considérablement leur mobilité par crainte d'être appréhendés par les policiers qui effectuent régulièrement des contrôles à l'entrée de la ville ou sur les routes principales autour de I'agglomération de Tyr. II devient alors encore plus difficile pour eux de trouver un emploi ou d'aller chercher l'aide humanitaire en centreville. Leurs conditions de logement se sont également dégradées, faute d'entretien de la part du propriétaire, alors qu'ils ont aujourd'hui un enfant de plus à charge. Cet exemple n'est pas isolé. Deux autres familles interrogées à Chabriha, qui ont pu bénéficier à leur arrivée d'une aide de la part de leurs proches, se sont vues contraintes de chercher un logement indépendant, leurs proches ne pouvant assumer les coûts induits par l'arrivée de familles souvent constituées d'au moins six personnes. 
La conséquence directe de cette paupérisation est que de nombreux réfugiés palestiniens du Liban tentent d'émigrer illégalement vers l'Europe. C'est le cas d'une jeune femme qui réside actuellement, avec ses deux enfants en bas âge, à Tyr dans une zone d'habitat informel à l'entrée nord de la ville. Elle partage son appartement avec trois autres familles. Elle espère rejoindre son frère qui vit à Malmö depuis neuf ans, marié avec une Palestinienne de nationalité suédoise. Son mari est parti en mars deTyr pour se rendre en Europe. II se trouve actuellement à Francfort, après un périple qui l'a fait passer par le Soudan, la Libye et I'Italie et lui a coûté 7000 euros. II attend de régulariser sa situation pour pouvoir faire venir sa famille, via le regroupement familial, et s'installer ensuite en Suède. Réunir cette somme a été très difficile. II a pu économiser un peu d'argent en travaillant au Liban pendant plus d'un an dans le bâtiment, et en empruntant à des proches, dont son beau-frère qui vit à Malmö. Ce type de tentative a un coût si prohibitif que l'on peut penser que d'un espace refuge, le Liban ne devienne une impasse pour ces réfugiés sans statut.

\section{Conclusion}

Alors que la majeure partie des réfugiés qui fuient la Syrie aujourd'hui est accueillie dans les pays limitrophes (Liban, Turquie, Jordanie et Irak) la situation des Palestiniens, plus problématique, interroge la définition du statut de réfugié dans un contexte hors convention (Erekat, 2014), alors qu'ils sont contraints de quitter leur pays de premier accueil, en l'occurrence la Syrie. L'expérience palestinienne permet de mener une réflexion sur les articulations entre migration forcée et circulation transfrontalière construites sur le long terme ; de nombreuses autres populations ayant été soumises à des expériences similaires dans la région (Chatty, 2010). Le rôle de la diaspora est aujourd'hui central dans I'accueil des réfugiés venus de Syrie. L'importance des réseaux diasporiques en situation de conflit n'est cependant pas nouvelle. La crise irakienne en 2003 avait réactivé des réseaux de la grande diaspora, une partie des Palestiniens d'Irak avaient obtenu I'asile au Chili, suite à la mobilisation de cette communauté pourtant installée dans ce pays dans la seconde moitié du XIXe siècle (Baeza, 2014). Les mouvements de contestation dans les pays arabes s'inscrivent dans leurs différents contextes nationaux, reléguant la question palestinienne à la marge. Cette marginalité a eu pour conséquence directe de priver de toute forme de protection les réfugiés palestiniens qui dépendent des politiques développées par leurs États d'accueil.

Le cas palestinien, malgré ses spécificités, pose la question des formes de solidarités particulières qui se réactivent en situation de conflit. Les solidarités familiales deviennent centrales pour certains groupes, même si elles ne sont pas exclusives, dans l'accueil des populations les plus défavorisées. Leur influence est décisive par exemple pour comprendre la géographie actuelle de l'asile palestinien au Liban et la poursuite du parcours vers des pays européens où de nombreux Palestiniens ont trouvé refuge depuis le milieu des années 1970. En l'absence de protection étatique ou internationale - I'UNRWA n'apportant qu'une assistance humanitaire -, les réfugiés palestiniens développent des stratégies migratoires qui leur permettent de contourner les restrictions dont ils sont l'objet. Si les réseaux de solidarités qui sont développés aux échelles locales espaces des camps et groupements au Liban - et transnationales - espaces de 
la diaspora - permettent de faire face en situation de crise, leur inscription dans la durée est beaucoup plus problématique, accroissant de fait les inégalités dans la société palestinienne entre ceux qui ont assez de ressources pour s'assurer un avenir stable en dehors de la région et les laissés-pour-compte confinés dans des espaces de relégation au Liban.

\section{Références bibliographiques}

Abou Zaki Hala C. (2015) Les réfugiés de Syrie dans le camp de Chatila : conflits de légitimité et solidarités entre "nouveaux" et " anciens " réfugiés, Confluences Méditerranée, 92, pp. 49-59. DOI : 10.3917/come.092.0049

Al-Natour Souheil (1997) The legal status of Palestinians in Lebanon, Journal of Refugee Studies, 10 (3), pp. 360-377.

Baeza Cecilia (2014) Palestinians in Latin America: Between Assimilation and Long-Distance Nationalism, Journal of Palestine Studies, 43 (2), pp. 59-72.

Brand Laurie (1988) Palestinians in Syria:The politics of integration, Middle East Journal, 42 (4), pp. 621-637.

Chaaban Jad, Ghattas Hala, Habib Rima, Hanafi Sari, Sahyoun Nadine, Salti Nisreen, Seyfert Karin and Naamani Nadia (2010) Socio-Economic Survey of Palestinian Refugees in Lebanon, American University of Beirut (AUB) and United Nations Relief and Works Agency for Palestine Refugees in the Near East (UNRWA), 90 p., [online]. URL: http://www.unrwa.org/userfiles/2011012074253. pdf

Chalcraft John (2009) The invisible cage, Syrian migrant workers in Lebanon, Stanford, California, Stanford University Press, 336 p.

Chatty Dawn (2010) Displacement and dispossession in the modern Middle East, vol. 5, Cambridge, Cambridge University Press, 335 p.

Doraï Kamel (2015) Palestinian refugees and the current Syrian conflict: from settled refugees to stateless asylum seekers ?, allegralaboratory.net, [online] last checked on 14/06/2015. URL: http://allegralaboratory.net/palestinian-refugees-and-the-current-syrian-conflict-from-settled-refugees-to-stateless-asylumseekers/

Doraï Kamel (2010) Palestinian Refugee camps in Lebanon. Migration, mobility and the urbanization process, in Are Knudsen and Sari Hanafi Eds., Palestinian Refugees. Identity, space and place in the Levant, London, Routledge, pp. 67-80.

Doraï Kamel (2006) Les réfugiés palestiniens au Liban : une géographie de l'exil, Paris, CNRS éditions, 256 p. (Coll. Moyen-Orient).

Doraï Kamel (2003) Palestinian Emigration from Lebanon to Northern Europe: Refugees, Networks, and Transnational Practices, Refuge (York University, Toronto), 21 (2), pp. 23-31.

Doraï Kamel et Al Husseini Jalal (2013) La vulnérabilité des réfugiés palestiniens à la lumière de la crise syrienne, Confluences Méditerranée, 87, pp. 95-108. 
Doraï Kamel et Hanafi Sari (2003) Des réfugiés qui ne relèvent pas du Haut Commissariat aux Réfugiés : les Palestiniens, in Michelle Guillon, Luc Legoux et Emmanuel Ma Mung Éds. L'asile politique entre deux chaises. Droits de I'Homme et gestion des flux migratoires, Paris, L'Harmattan, pp. 287-310.

Dot-Pouillard Nicolas (2012) Les révolutions arabes entre césures et remembrances : tiers-mondisme, question palestinienne et utopies chiliastiques, L'Année du Maghreb, 8 [en ligne], consulté le 03/09/2015. URL : http://anneemaghreb. revues.org/1393 - DOI : 10.4000/anneemaghreb.1393

Erakat Noura (2014) Palestinian Refugees and the Syrian Uprising: Filling the Protection Gap during Secondary Forced Displacement, International Journal of Refugee Law, 26 (4), pp. 581-621.

Fadhel Khadija (2012) Le camp de Jaramana, Un espace marginal au cœur de l'agglomération damascène, in Kamel Doraï et Nicolas Puig Éds., L'urbanité des marges : migrants et réfugiés dans les villes du Proche-Orient, Paris, Téraèdre, pp. $115-138$.

Kagan Michael (2011) "We live in a country of UNHCR". The UN surrogate state and refugee policy in the Middle East, UN High Commissioner for Refugees (UNHCR), 25 p., [online] last check on 08/03/2015. URL: http://www.refworld.org/ docid/4d8876db2.html

Kagan Michael (2009) Is there Really a Protection Gap? UNRWA's Role vis-à-vis Palestinian, Refugees Refugee Survey Quarterly, 28 (2-3), pp. 511-530

Knudsen Are J. (2013) Violence et déplacement : la crise des réfugiés syriens au Liban, Maghreb - Machrek, 4 (218), pp. 29-40. DOI : 10.3917/machr.218.0029

Longuenesse Élisabeth (2015) Travailleurs étrangers, réfugiés syriens et marché du travail, Confluences Méditerranée, 92, pp. 33-47. DOI : 10.3917/come.092.0033

Malkki Liisa H. (1995) Refugees and Exile: From "Refugee Studies" to the National Order of Things, Annual Review of Anthropology, 24, pp. 495-523.

Mohanna Kamel et Micheletti Pierre (2014) Liban-Syrie : solidarité et business, Esprit, 10, pp. 127-129.

Napolitano Valentina (2015) Militant Conversion and Transformation of Palestinian Civil Society Organizations in al-Yarmouk, Al Majdal, 57, Bethléem, Badil Resource Centre, pp 11-15.

Napolitano Valentina (2012) La mobilisation des réfugiés palestiniens dans le sillage de la " révolution " syrienne : s'engager sous contrainte, Cultures \& conflits, 87, pp. 119-137.

Napolitano Valentina (2011) Les réfugiés palestiniens et la contestation populaire en Syrie, Esprit, 7, pp. 169-174.

Smith Michael P. (2002) Preface, in Nadje Al-Ali and Khalid Koser Eds., New Approaches to Migration? Transnational Communities and the Transformation of Home, London, Routledge, pp. 11-15.

Takkenberg Lex (1998) The Status of Palestinian Refugees in International Law, Oxford, Clarendon Press, 411 p.

Van Hear Nicholas (2014) Reconsidering Migration and Class, International Migration Review, 48 (S1), pp. 100-121. 
Van Hear Nicholas (2006) Refugees in diaspora: From durable solutions to transnational relations, Refuge, 23 (1), pp. 9-15.

Wahlbeck Östen (2002) The concept of diaspora as an analytical tool in the study of refugee communities, Journal of Ethnic and Migration Studies, 28 (2), pp. 221-238.

Zaiotti Ruben (2006) Dealing with Non-Palestinian Refugees in the Middle East: Policies and Practices in an Uncertain Environment, International Journal of Refugee Law, 18 (2), pp. 333-353.

Zureik Elia (2003) Theoretical and methodological considerations for the study of Palestinian society, Comparative Studies of South Asia, Africa and the Middle East, 23 (1), pp. 152-162. 


\section{Kamel Doraï}

\section{Les Palestiniens et le conflit syrien. Parcours de réfugiés en quête d'asile au Sud-Liban}

Depuis 2011, le conflit syrien a donné naissance à l'un des mouvements de réfugiés les plus massifs au Moyen-Orient, depuis la Seconde Guerre mondiale. Comme de nombreux Syriens, les réfugiés palestiniens de Syrie, ont fui les combats et se sont installés dans les pays frontaliers. Ce mouvement a été largement occulté par l'ampleur de la crise syrienne et la masse des 4 millions de réfugiés syriens. À partir de deux séries d'enquêtes réalisées dans les camps et groupements palestiniens autour de la ville de Tyr en 2013 et 2015, cet article a pour objectif de rendre compte de la diversité des expériences migratoires des Palestiniens de Syrie réfugiés au Liban et de montrer comment ils ont pu mobiliser des réseaux de solidarité qui se sont développés grâce aux mobilités transfrontalières et à la structuration de la diaspora palestinienne.

\section{The Palestinians and the Syrian Conflict. Itineraries of Refugees Seeking Asylum in South Lebanon}

The Syrian conflict that began in 2011 has generated one of the most massive refugee movements since the Second World War in the Middle East. Palestinian refugees in Syria, among the least discriminated group in terms of the treatment they may experience in other countries in the region, saw themselves sent back to their stateless status, without any protection and forced to seek asylum in neighbouring countries. This movement largely overshadowed by the magnitude of the Syrian crisis, triggered by the conflict in Syria since 2011, has reactivated solidarity networks that have developed through cross-border circulation and structured by the Palestinian Diaspora. Based on two series of interviews conducted in the Palestinian camps and gatherings around the city of Tyre in 2013 and 2015, this article aims to reflect the diversity of migratory experiences of Syrian Palestinians seeking asylum in Lebanon.

\section{Los palestinos y el conflicto sirio. Itinerarios de refugiados solicitando asilo en el sur del Líbano}

El conflicto sirio, que empezó en 2011, ha generado uno de los movimientos de refugiados más masivos desde la Segunda Guerra Mundial en el Medio Oriente. Los refugiados palestinos en Siria, entre el grupo menos discriminado en los países de la zona, se vieron devueltos a su condición apátrida, sin ningún tipo de protección, y forzado a buscar asilo en los países vecinos. Este movimiento, eclipsado por la magnitud de la crisis siria, si se activa tras el conflicto en Siria desde 2011, ha reactivado las redes de solidaridad que se han desarrollado a través de la movilidad transfronteriza y la estructuración de la diáspora palestina. A partir de dos series de encuestas realizadas en los campos palestinos y grupos alrededor de la ciudad de Tiro, en 2013 y 2015, este artículo tiene como objetivo reflejar la diversidad de las experiencias migratorias Palestinos de Siria buscando asilo en Líbano. 